\title{
Conocimientos de higiene oral de acudientes y su relación con caries en menores de 5 años
}

\author{
Parent's Knowledge about Oral Hygiene and its Relation with Cavities \\ in Children under 5 Years of Age
}

\author{
Conhecimentos sobre higiene oral que os adultos responsáveis \\ de crianças têm e a sua relação com as cáries em infantes com \\ menos de 5 anos
}

\author{
José Julián Rodríguez-Álvarez', Dedsy Yajaira Berbesí-Fernández² \\ 1 Magíster en epidemiologia. Odontólogo, ESE Metrosalud, Colombia. jotaroal@yahoo.com, orcid.org/0000-0002- \\ 2677-7569 \\ 2 Doctora en epidemiologia y bioestadística. Enfermera, Universidad cEs, Colombia. dberbesi@ces.edu.co.
}

Recibido: 18/06/2015. Aprobado: 08/05/2018. Publicado: 30/04/2018

Rodríguez-Álvarez JJ, Berbesí-Fernández DJ. Conocimientos de higiene oral de acudientes y su relación con caries en menores de 5 años. Rev. Fac. Nac. Salud Pública.2018;36(2):7-17. DoI:10.17533/udea.rfnsp.v36n2a03

\section{Resumen}

Objetivo: Identificar los conocimientos en higiene oral que poseen los acudientes y su relación con la presencia de caries dental de niños menores de 5 años que asisten a un centro de salud de Medellín. Metodología: Se realizó un estudio de casos y controles. El grupo de casos estuvo conformado por los acudientes, cuyos niños tuvieran un índice ceo-d mayor que cero. El grupo control lo conformaron los acudientes cuyos niños tuvieron un índice ceo-d igual a cero. Las fuentes de información fueron la valoración odontológica para determinar la existencia o no de caries cavitacional, la historia clínica y un instrumento sobre los conocimientos de higiene de los acudientes. Se analizó origen, apariencia y formas de prevención de la caries. Resultados: La presencia de caries dental estuvo asociada con falta de control de la cantidad de crema dental por parte de los acudientes, porque creían que dejaba más limpios los dientes (OR 4,29; IC $95 \%$ : 1,7410,62 ), porque creían que no hacía daño (OR 3,29; IC $95 \%$ : 1,61-6,75). Conclusiones: se identificó la asociación entre las limitaciones en el conocimiento que sobre higiene oral tienen los acudientes y la existencia de caries dental en relación con la apariencia de la caries y el uso de la crema dental en los niños de la muestra. Los resultados sugieren la necesidad de generar espacios de comunicación e interacción entre los profesionales de la salud y los usuarios, acciones educativas oportunas que generen impacto, la utilización de medios de comunicación y diferentes estrategias de información, educación y comunicación (IEC) que permitan una mayor aprehensión del conocimiento.

-Palabras clave: caries dental, higiene oral, salud oral, promoción de la salud, educación sanitaria. 


\section{Abstract}

Objective: to identify parent's knowledge about oral hygiene and its relation with dental cavities in their children under 5 years of age, patients of a health center in Medellin. Methodology: Case and control study. The group of cases was composed by parents whose children had a DMFT index higher than zero. The control group was composed by parents whose children had a DMFT index equal to zero. Information was collected through dental evaluations, in order to determine the presence of cavities, clinic records and a document with parents' knowledge about dental hygiene. The origin, appearance and way to prevent the cavities were analyzed Results: the presence of cavities was associated with lack of control of the amount of toothpaste on the parents' side, because they believed the teeth were kept cleaner (OR 4,29; IC $95 \%$ : 1,74-10,62), because they did not find it harmful (OR 3,29; IC $95 \%$ : 1,61-6,75). Conclusions: We identified an association between parents' poor knowledge about oral hygiene, the appearance of cavities and toothpaste usage in the sampled children. The results suggests a need to create communication and interaction spaces between health professionals and the users, and timely educational actions that could have an impact, using mass media and other information, education and communication (IEC) strategies that allow a better appropriation of knowledge.

--------Key words: dental cavity, oral hygiene, oral health, health promotion, sanitary education.

\section{Resumo}

Objetivo: identificar os conhecimentos sobre higiene oral que têm os adultos responsáveis de crianças e a sua relação com a aparição de cáries dentais em infantes com menos de 5 anos, pacientes de um centro de saúde em Medellín. Metodologia: realizou-se um estudo de casos e controles. O grupo de casos esteve conformado pelos adultos responsáveis, cujas crianças tiveram um índice ceo-d mais alto do que cero. As fontes de informação foram a valoração odontológica para determinar a existência ou não de cáries cavitacional, a história clínica e um instrumento sobre os conhecimentos de higiene dos adultos responsáveis de crianças. Analisou-se a origem, aparência e forma de prevenção de cáries. Resultados: a presencia de cáries dentais associou-se com falta de controle da quantidade de creme dental por parte dos adultos responsáveis, porque achavam que os dentes ficavam mais limpos (OR 4,29; IC 95 \%: 1,74-10,62), porque achavam que não era nocivo (OR 3,29; IC 95 \%: 1,61-6,75). Conclusões: identificou-se a associação entre as limitações no conhecimento que tem os adultos responsáveis sobre higiene oral e a existência de cáries dentais em relação com a aparência da cárie e o uso do creme dental nas crianças da amostra. Os resultados sugerem a necessidade de gerar espaços de comunicação e de interação entre os profissionais de saúde e os usuários, ações educativas oportunas que gerem impacto, a utilização de meios de comunicação e diferentes estratégias de informação, educação e comunicação (IEC) que permitam mais apropriação do conhecimento.

--------Palavras-chave: cárie dental, higiene oral, saúde oral, difusão da saúde, educação sanitária.

\section{Introducción}

A pesar de los grandes avances de la ciencia y la implementación continua de programas que fomentan la práctica de hábitos benéficos para la salud humana, la caries dental aparece como una de las enfermedades infecciosas más prevalentes y más difíciles de erradicar. En Estados Unidos, más del $40 \%$ de los niños entre 2 y 11 años de edad presentaron lesiones clínicas detectables de caries en los dientes deciduos [1]. En la India, en el año 2013, se encontró que la prevalencia de caries en niños de 3 años era del 44,3 \% [2]. El predominio de esta enfermedad en países industrializados, con la misma ocurrencia que en naciones en proceso de desarrollo, y donde el acceso a los servicios de salud es más alcanzable, lleva a pensar que el problema de la caries está más relacionado con la forma como se percibe esta enfermedad dentro de la comunidad expuesta, que con la disponibilidad que se tenga de ciertos recursos económicos o de acceso a los servicios de salud [1]. En Colombia, es una enfermedad que afecta a más de la mitad de la población.

Se ha logrado determinar, a través de los años y por múltiples investigaciones, que la caries dental es una patología multifactorial que se manifiesta en la cavidad oral como lesión de origen infeccioso, que inicia con cambios en la microflora bacteriana de dicha cavidad, en la que se involucran el flujo y la composición de la saliva, el consumo de alimentos azucarados, la exposición al fluoruro y las medidas preventivas, como limpiarse los dientes [3].

De igual manera, se convierte en una enfermedad que produce daños irreparables, dolor e incomodidad, además de que se reduce la ingesta de alimentos, porque comer se convierte en una actividad dolorosa [4]. Igualmente, la caries puede ocasionar alteraciones en el bienestar general de los niños, causándoles irritabilidad y trastornos en el sueño $[5,6]$. 
Por causa de la caries dental pueden manifestarse síntomas de dolor cuya intensidad no solo dependerá del huésped, sino también del grado de extensión y severidad de la lesión [7]. Cuando no se trata oportunamente la caries en los niños, aumenta el riesgo de aparición de otras enfermedades sistémicas y complicaciones de tipo infeccioso [8].

Existen una serie de factores externos, como los estilos de vida, la cultura, la edad, costumbres familiares y el entorno sociocultural, que tienen influencia de forma indirecta en la aparición de enfermedades de tipo crónico como la caries dental [9].

Los padres de familia son los primeros educadores de sus hijos en edades tempranas; por esta razón, es importante conocer qué saben aquellos acerca del autocuidado, para, de esta manera, poder descubrir si es necesario reforzar algún concepto deficiente, que permita empoderarlos como acompañantes significativos en el proceso de desarrollo de sus hijos [10].

En Colombia, las actividades en las instituciones de salud se enfocan mucho más en el tratamiento de la enfermedad y muy poco en la prevención [11,12]; además, se conocen pocos estudios que muestren qué saben las personas acerca del buen mantenimiento de la cavidad oral y su relación con la caries, similar a lo observado en el año 2010 en Perú [13].

El enfoque del estudio en la población menor de 5 años refleja la preocupación de encontrar índices de caries dental elevados, sabiendo que es una enfermedad prevenible, en un grupo etario que tiene completa dependencia de otra persona y que se encuentra indefensa ante cualquier situación que comprometa su salud. En la presente investigación se buscó establecer si los conocimientos de higiene oral con que cuentan los acudientes influyen en la aparición de la caries dental en los menores de 5 años.

\section{Metodología}

Se realizó un estudio de casos y controles con muestreo no probabilístico. Se hicieron exámenes odontológicos a los niños menores de 5 años que asistieron a un centro de salud de Medellín, para determinar la existencia o no de caries cavitacional, mediante la aplicación del índice ceo-d. Se incluyó una revisión de historia clínica, para verificar antecedentes y enfermedades de base, con el fin de confirmar si cumplían los criterios para ser incluidos en el estudio.

El índice ceo-d fue adoptado por Allen Gruebbel en 1944. En él se verifican los dientes deciduos cariados, con extracciones indicadas por caries y obturados por caries. El valor mínimo del índice es 0 y el máximo es 20 [14].
Los criterios de inclusión para este estudio fueron: los menores de 5 años que consultaron al servicio de odontología en un centro de salud de la Empresa Social del Estado (ESE) Metrosalud, y los acudientes, mayores de 18 años, responsables de aquellos, que accedieron a participar en el estudio. Los criterios de exclusión fueron: los niños menores de 5 años con registro en la historia clínica de enfermedades autoinmunes, cáncer, tratamiento de radio- o quimioterapia, niños menores de 5 años con dentición mixta y los cuidadores que no dieron el consentimiento para participar en la investigación.

Se calculó un tamaño de muestra de 87 casos y 87 controles en el programa Epidat 3.1 [15], teniendo en cuenta los siguientes criterios:

- Un nivel de confianza del $95 \%(\alpha=0,05)$.

- Un poder del $80 \%(\beta=0,2)$, teniendo en cuenta una proporción de casos expuestos a bajos conocimientos de higiene oral de $83 \%$ y de controles expuestos a bajos conocimientos de higiene oral del $63 \%$, con un Odds Ratio (OR) esperado de 2,87 [1]. El grupo de casos estuvo conformado por los acudientes cuyos niños tuvieran un índice ceo-d mayor que cero.

Luego, a cada acudiente se le aplicó el instrumento desarrollado por Martignon et al., para medir los conocimientos de higiene oral en cada uno de ellos, que constaba de 24 preguntas con 74 ítems. Esta encuesta fue validada por Martignon et al. en la localidad de Usaquén, en la ciudad de Bogotá, Colombia, aplicándola en padres de familia de niños menores de 5 años de estrato socioeconómico bajo [16], una población con condiciones similares a los participantes del presente estudio. También se determinaron las características sociales y demográficas de los pacientes menores de 5 años y sus acudientes.

El procesamiento y el análisis de información se desarrolló en el paquete estadístico SPSS ${ }^{\circledR}$ (versión portable PASW Statistics 18, sPSS Inc. Chicago, IL).

Se calcularon las proporciones de las diferentes variables sociodemográficas de los acudientes de los niños menores de 5 años. Para determinar la relación entre los conocimientos de higiene oral con que cuentan aquellos y la caries dental de los menores de 5 años bajo su cargo, se efectuó un análisis multivariado de regresión logística binaria, utilizando el método "Introducir". Se calcularon los or ajustados para cada variable con sus intervalos de confianza al $95 \%$. Un valor $p \leq 0,05$ fue considerado significativo.

Así mismo, se emplearon medidas estadísticas para las pruebas de hipótesis, de acuerdo con la naturaleza y la distribución (normalidad) de las variables, para establecer la existencia de diferencias significativas entre los grupos; variables continuas, con la prueba $t$ de Student (normales), o la prueba con signos de Wilcoxon (variables no normales). La prueba exacta de Fisher o 
la prueba de chi-cuadrado se emplearon para comparar variables categóricas.

Se obtuvo el aval del Comité de Investigaciones de la eSE Metrosalud (en oficio escrito del día 2 de diciembre de 2013). Los acudientes aceptaron proporcionar la información que se requirió, mediante la firma del consentimiento informado.

De acuerdo con la Resolución 8430 de 1993 de la República de Colombia, expedida por el Ministerio de Salud, y tal como dicta en el Título II, capítulo 1, artículo 11 [17], esta fue considerada una investigación sin riesgo, cuya intervención se limitó a la recolección y el análisis de información clínica y demográfica de los pacientes que asistieron a consulta odontológica en un centro de salud de la ciudad de Medellín.
Se garantizó en todo momento la protección de la privacidad de los pacientes sujetos de estudio y se espera que los resultados puedan ser usados en la implementación de medidas de salud pública que beneficien a la comunidad.

\section{Resultados}

\section{Variables sociodemográficas}

De las 174 personas encuestadas, el $89 \%$ se identificaron como las madres de los niños examinados. Además, el $69 \%$ informó haber estudiado solamente hasta la educación básica primaria (véase figura 1).

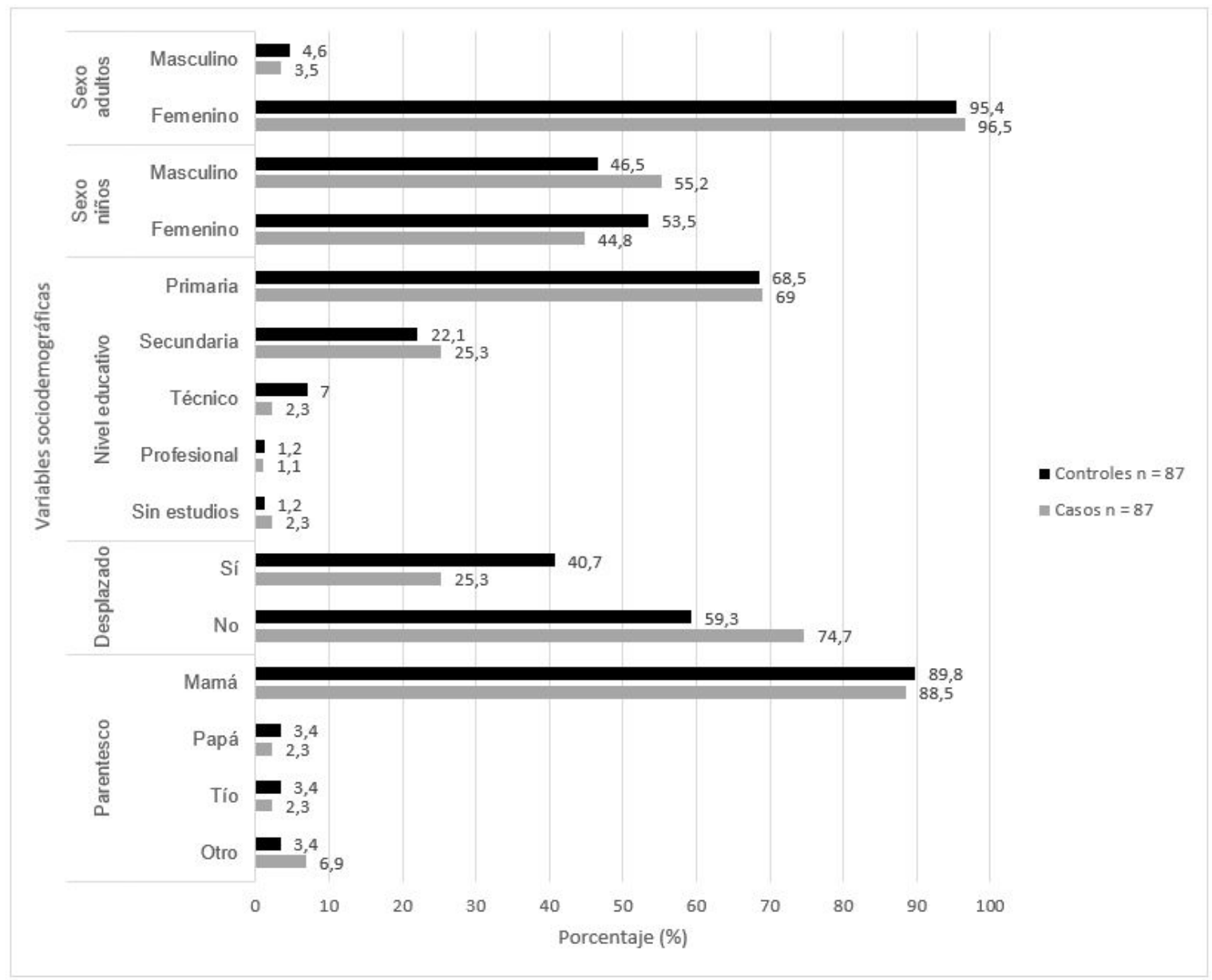

Figura 1. Características sociodemográficas de niños menores de 5 años (valores dados en porcentaje). 


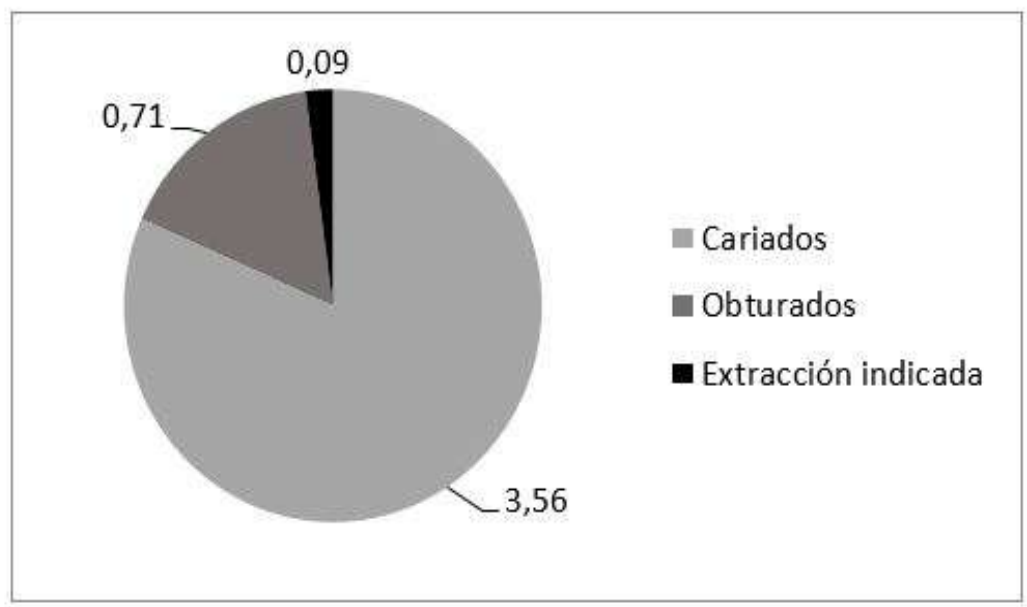

Figura 2. Promedio de dientes cariados, obturados y con extracción indicada por caries.

El promedio de dientes cariados fue de 3,5 dientes (Desviación estándar $-\mathrm{DE}-=3,5$ ), así como la media del índice ceo-d en los niños menores de 5 años fue 4,3 dientes $(\mathrm{DE}=3,4)$ (véase figura 2$)$.

El rango intercuartil (RIq) de la edad de los acudientes en el grupo casos es 13 , con una mediana de 28 , mientras que en el grupo controles el RIq es 12 , con una mediana de 23, mostrando una dispersión similar en esta categoría en ambos grupos. En la edad de los niños evaluados, el grupo casos tuvo una mediana de 4 y el grupo controles una mediana de 1; para ambos grupos, el RIq tuvo un valor de 2.

$\mathrm{Al}$ evaluar la distribución de la caries dentro del estudio, se observa que a medida que aumenta la edad de los niños evaluados, también crece la prevalencia de la caries dental. En los niños de 1 año es del $2 \%$ y en los de 5 años aumenta al $36 \%$ (véase tabla 1 ).

Tabla 1. Distribución por edades de dientes ceo.

\begin{tabular}{lccccc}
\hline \multirow{2}{*}{ Edad } & \multicolumn{6}{c}{ Casos $\mathbf{n}=\mathbf{8 7}$} \\
\cline { 2 - 6 } & $\mathbf{n}$ & $\%$ & $\mathbf{c}$ & $\mathbf{e}$ & $\mathbf{0}$ \\
\hline 1 & 2 & 2,3 & 5 & 0 & 0 \\
2 & 11 & 12,6 & 33 & 2 & 3 \\
3 & 25 & 28,7 & 92 & 14 & 0 \\
4 & 18 & 20,7 & 78 & 9 & 4 \\
5 & 31 & 35,6 & 102 & 37 & 1 \\
\hline Total & 87 & 100 & 310 & 62 & 8 \\
\hline Los valores del índice ceo son mínimo 0 máximo 20. \\
c: dientes deciduos cariados; e: dientes con extracciones indicadas por caries; o: obturados \\
por caries.
\end{tabular}

\section{Conocimientos en higiene oral}

Los padres de familia son los primeros educadores de sus hijos en edades tempranas; por esta razón, es importante conocer sus saberes acerca del mantenimiento de un cuerpo saludable y de esta manera poder descubrir si es necesario reforzar o reformular algún concepto deficiente que permita hacer de los adultos respon- sables un instrumento útil en el proceso de desarrollo de sus hijos [10].

Origen de la caries

Al hacer la estimación del riesgo de caries a partir de los conocimientos que de higiene oral poseen los acudientes, se encontró que es 1,2 veces más probable que un niño tenga caries si la persona que lo cuida no cree que 
aquella se produce por una mala alimentación $(\mathrm{OR}=1,2$; IC $95 \%=0,7-2,3)$. La probabilidad de que a un niño menor de 5 años tenga caries es dos veces más alta en aquellos cuyos acudientes no aseguren que la caries se produce por una mala higiene oral, que en los que así lo crean $(\mathrm{OR}=2$; IC $95 \%=0,1-22,4)$.

Los infantes cuyos tutores no sabían que la caries dental inicialmente se ve como una mancha blanca tienen 3,3 más probabilidades de tener caries, que los niños cuyos acudientes afirmaron conocer la apariencia inicial de la caries $(\mathrm{OR}=3,3$; IC $95 \%=0,9-11,5)$.

Los niños de quienes sus tutores no sabían cómo evitar el progreso de la caries dental cuando esta ya ha hecho una cavidad tienen 2,5 más probabilidades de generar caries dental, que aquellos menores cuyos acudientes conocían las actividades para evitar el avance de la lesión cavitaria ( $\mathrm{OR}=2,5$; IC $95 \%=1,3-5,0)$.

\section{Visita al odontólogo y cuidado de los dientes deciduos}

Del mismo modo, aun cuando no se encontró significación estadística, fue dos veces más alta la probabilidad de la aparición de caries en los infantes cuyos acudientes no aseguraron que la forma más efectiva de evitar la caries es asistiendo al odontólogo, que en los que sí lo afirmaron $(\mathrm{OR}=2,2 ;$ IC $95 \%: 0,9-5,2)$. Fue también un factor protector de la caries en los infantes, el que los entrevistados expresaran que se debía cuidar los dientes de leche, para que los niños tuvieran una sonrisa bonita $(\mathrm{OR}=0,9$; IC $95 \%=0,4-2,1)$ y para que tuvieran una buena salud $(\mathrm{OR}=0,7$; IC $95 \%=0,2-2,1)$. Además, fue factor de protección que los acudientes confirmaran que deben cuidarse los dientes de leche para que los niños puedan comer y hablar bien ( OR $=0,4$; IC $95 \%=0,1-1,2)$.

Es 2,4 veces mayor la posibilidad de tener caries para aquellos niños cuyos acudientes no consideraron importante cuidar los dientes de leche, porque se dañaban fácil, que para quienes sus responsables sí tuvieron en cuenta que era importante cuidar los dientes deciduos.

\section{Higiene oral}

Careció de significación estadística recibir información acerca del cuidado de higiene oral ( $\mathrm{OR}=0,6$; IC $95 \%=0,3-1,2)$. En quienes recibieron esta información por parte del odontólogo ( OR $=0,6$; IC $95 \%=0,3$ $1,2)$, el riesgo fue menor, que si es recibida por parte del médico $(\mathrm{OR}=0,9$; IC $95 \%=0,5-1,8)$ o por la profesora de guardería (OR $=0,7$; IC $95 \%=0,3-1,6)$.

Confirmar que la limpieza de la cavidad oral de los niños debe empezarse desde el nacimiento o cuando comienza la erupción de los dientes deciduos, fue un factor no significativo contra la caries dental $(\mathrm{OR}=0,8$; IC $95 \%=0,4-1,5)$. Aquellos niños cuyos acudientes expusieron que la limpieza de la boca se comienza al completar la dentición decidua tienen 1,3 veces más riesgo de tener caries, que los niños cuyos acudientes comienzan la higiene de la cavidad oral con mayor anterioridad $(\mathrm{OR}=1,3$; IC $95 \%=0,4-4,0)$.

Los infantes cuyos acudientes confirmaron que era importante realizar higiene oral para evitar la formación de caries $\mathrm{y}$, además, crear la costumbre de higiene, tienen menor riesgo de caries, que aquellos cuyos acudientes respondieron negativamente acerca de la importancia de la higiene oral ( $\mathrm{OR}=0,5$; IC $95 \%=0,2-1,0)$. Los niños cuyos acudientes consideraron que no era importante hacer higiene oral en los infantes tienen 1,6 más probabilidades de tener caries, que los niños cuyos tutores respondieron de manera afirmativa (OR $=1,6$; IC $95 \%=0,8-3,1)$.

\section{Crema dental}

Considerar que la crema dental es importante en la higiene oral fue factor de protección no significativo contra la caries dental en los niños cuyos tutores contestaron afirmativamente a este cuestionamiento $(\mathrm{OR}=0,6$; IC $95 \%=0,3-1,4)$.

Los menores cuyos acudientes no controlan la cantidad de crema dental porque no hace daño, tienen 3 veces más riesgo de tener caries, que los niños a quienes sus acudientes ejercen control ( $\mathrm{OR}=3,2$; IC $95 \%=1,5-6,5)$. Entre tanto, los niños para los que sus acudientes consideraron que la cantidad ideal de crema dental a utilizar sí actúa como factor protector y era como el tamaño de la uña del dedo meñique de un adulto, cuentan con menor riesgo de tener caries, que aquellos niños donde sus tutores eligieron otra cantidad $(\mathrm{OR}=0,7$; IC $95 \%=0,2-2,1)$.

\section{Flúor}

Aquí se investigó el conocimiento de los acudientes de para qué sirve el flúor. Hubo asociación entre tener caries y que los acudientes supieran que el flúor servía para prevenir y tratar la caries dental $(\mathrm{OR}=0,8$; IC $95 \%=0,4-1,6)$, aunque no fue estadísticamente significativo. Del mismo modo, se presentó asociación entre el conocimiento que tienen los acudientes de que el uso de crema dental y enjuagues con flúor podría detener el progreso de la caries y aumentar la protección contra la caries $(\mathrm{OR}=0,7$; IC $95 \%=0,4-1,4)$.

Luego de realizar un análisis multivariado de regresión logística binaria, se calcularon los or ajustados, con sus respectivos intervalos de confianza, considerando significativo un valor de $p \leq 0,05$ (véase tabla 2).

\section{Discusión}

Distintos investigadores han hecho varias búsquedas para describir la prevalencia de caries en niños en edad preescolar $[8,18,19]$; sin embargo, no se había explorado una relación de los conocimientos que poseen los acudientes 
Tabla 2. Conocimientos de los acudientes de los menores de 5 años acerca de cómo cuidar la higiene oral.

\begin{tabular}{|c|c|c|c|c|c|c|c|c|c|c|}
\hline \multirow{3}{*}{$\begin{array}{l}\text { Características } \\
\text { Conocimientos }\end{array}$} & & \multicolumn{4}{|c|}{ Caries } & & & \multirow{3}{*}{$\begin{array}{l}\text { OR crudo } \\
\text { (Ic } 95 \% \text { ) }\end{array}$} & \multirow{3}{*}{$\begin{array}{l}\text { OR ajustado } \\
\text { (IC } 95 \% \text { ) }\end{array}$} & \multirow{3}{*}{ Valor $p$} \\
\hline & & \multicolumn{2}{|c|}{ Casos $(n=87)$} & \multicolumn{2}{|c|}{ Controles $(n=87)$} & \multicolumn{2}{|c|}{ Total } & & & \\
\hline & & $\mathrm{n}$ & $\%$ & $\mathrm{n}$ & $\%$ & $\mathrm{n}$ & $\%$ & & & \\
\hline \multirow{2}{*}{$\begin{array}{l}\text { La mejor manera } \\
\text { de evitar la caries } \\
\text { dental es ir al } \\
\text { odontólogo }\end{array}$} & No & 18 & 20,7 & 9 & 10,3 & 27 & 15,5 & \multirow[b]{2}{*}{$2,20(0,9-5,2)$} & \multirow[b]{2}{*}{$0,60(0,09-4,0)$} & \multirow[b]{2}{*}{0,06} \\
\hline & Sí & 69 & 79,3 & 78 & 89,7 & 146 & 84,5 & & & \\
\hline \multirow{2}{*}{$\begin{array}{l}\text { Recibió información } \\
\text { de cuidado dental } \\
\text { para el niño }\end{array}$} & No & 24 & 27,6 & 33 & 37,9 & 56 & 32,8 & \multirow{2}{*}{$0,60(0,3-1,2)$} & \multirow{2}{*}{$1,87(0,42-8,23)$} & \multirow{2}{*}{0,17} \\
\hline & Sí & 63 & 72,4 & 54 & 62,1 & 117 & 67,2 & & & \\
\hline \multirow{2}{*}{$\begin{array}{l}\text { Importante realizar } \\
\text { higiene oral en el } \\
\text { niño para evitar } \\
\text { caries }\end{array}$} & No & 20 & 23,0 & 11 & 12,6 & 31 & 17,8 & \multirow[b]{2}{*}{$2,0(0,9-4,5)$} & \multirow[b]{2}{*}{$9,60(1,7951,45)$} & \multirow[b]{2}{*}{0,08} \\
\hline & Sí & 67 & 77,0 & 76 & 87,4 & 142 & 82,2 & & & \\
\hline \multirow{2}{*}{$\begin{array}{l}\text { Importante realizar } \\
\text { higiene oral en el } \\
\text { niño para evitar } \\
\text { caries y crear } \\
\text { costumbre }\end{array}$} & No & 18 & 20,7 & 29 & 33,3 & 47 & 27,0 & \multirow[b]{2}{*}{$0,50(0,2-1,0)$} & \multirow[b]{2}{*}{$1,95(0,98-3,86)$} & \multirow[b]{2}{*}{0,08} \\
\hline & Sí & 69 & 79,3 & 58 & 66,7 & 126 & 73,0 & & & \\
\hline \multirow{2}{*}{$\begin{array}{l}\text { No es importante } \\
\text { realizar higiene oral }\end{array}$} & No & 30 & 34,5 & 22 & 25,3 & 51 & 29,9 & \multirow{2}{*}{$1,60(0,8-3,1)$} & \multirow{2}{*}{$0,61(0,31-1,18)$} & \multirow{2}{*}{0,20} \\
\hline & Sí & 57 & 65,5 & 65 & 74,7 & 122 & 70,1 & & & \\
\hline \multirow{2}{*}{$\begin{array}{l}\text { No controla la } \\
\text { cantidad de crema } \\
\text { dental, porque no } \\
\text { hace daño }\end{array}$} & No & 34 & 39,1 & 14 & 16,1 & 48 & 27,6 & \multirow[b]{2}{*}{$3,20(1,5-6,5)$} & & \\
\hline & Sí & 53 & 60,9 & 73 & 83,9 & 125 & 72,4 & & $3,29(1,61-6,75)$ & $0,001^{*}$ \\
\hline $\begin{array}{l}\text { No controla la } \\
\text { cantidad de crema }\end{array}$ & No & 24 & 27,6 & 7 & 8,0 & 31 & 17,8 & & & \\
\hline $\begin{array}{l}\text { dental, porque a } \\
\text { más cantidad, más } \\
\text { limpios quedan }\end{array}$ & Sí & 63 & 72,4 & 80 & 92,0 & 142 & 82,2 & $4,00(1,6-9,8)$ & $4,29(1,74-0,62)$ & $0,001^{*}$ \\
\hline La cantidad de & No & 28 & 32,2 & 16 & 18,4 & 43 & 25,3 & & & \\
\hline $\begin{array}{l}\text { crema dental a usar } \\
\text { debe ser la mitad } \\
\text { del largo del cepillo }\end{array}$ & Sí & 59 & 67,8 & 71 & 81,6 & 130 & 74,7 & $2,20(1,0-4,4)$ & $2,03(0,45-9)$ & 0,11 \\
\hline Sabe para qué se & No & 66 & 75,9 & 57 & 65,5 & 123 & 70,7 & $150(08-30)$ & $062(032-121)$ & 022 \\
\hline & Sí & 21 & 24,1 & 30 & 34,5 & 50 & 29,3 & (1, & $0,0<(0,0<-1,<1)$ &, ,LC \\
\hline Lesión inicial de & No & 84 & 96,6 & 77 & 88,5 & 160 & 92,5 & & & \\
\hline $\begin{array}{l}\text { carles se ve como } \\
\text { mancha blanca en el } \\
\text { diente }\end{array}$ & Sí & 3 & 3,4 & 10 & 11,5 & 13 & 7,5 & $3,30(0,9-11,5)$ & $2,93(0,74-11,5)$ & 0,12 \\
\hline Sabe cómo evitar & No & 57 & 65,5 & 47 & 54,0 & 103 & 59,8 & & & \\
\hline $\begin{array}{l}\text { progreso de lesión } \\
\text { de caries }\end{array}$ & Sí & 30 & 34,5 & 40 & 46,0 & 70 & 40,2 & $1,60(0,8-3,0)$ & $0,60(0,32 ; 1,11)$ & 0,14 \\
\hline No sabe cómo es & No & 45 & 51,7 & 40 & 46,0 & 85 & 48,9 & & & \\
\hline $\begin{array}{l}\text { daño dental donde } \\
\text { hay hueco }\end{array}$ & Sí & 42 & 48,3 & 47 & 54,0 & 88 & 51,1 & $2,50(1,3-5,0)$ & $4,24(1,12-15,9)$ & 0,07 \\
\hline
\end{tabular}

* Valores significativos en las pruebas estadísticas $(p<0,05)$.

OR: Odds Ratio; IC: Intervalo de confianza.

en higiene oral, con la caries dental de los niños, en un estudio de casos y controles.

En esta investigación se identificaron los conocimientos que de higiene oral poseen adultos cuidadores de niños menores de 5 años y su relación con la presencia de caries dental en una comuna de la ciudad de Medellín, encontrando factores que pueden orientar intervenciones y acciones preventivas en el área de salud oral.

El promedio del índice ceo-d muestra la severidad de la caries en los niños menores de 5 años 
que se encontraban dentro de los casos. Este tuvo un valor superior con respecto al hallado en el IV Estudio Nacional de Salud Bucal (ENSAB IV), hecho en Colombia en 2014, que reveló un índice de severidad en promedio de 1,55 dientes, es decir, que dos niños en promedio tienen tres dientes con caries [20]. La mayor presencia de caries en los niños estudiados en la comuna de Medellín puede sugerir un menor nivel de conocimientos sobre la prevención y el cuidado de la cavidad oral.

Aseguró Saied-Moallemi [18], en un estudio hecho en Irán en 2006, que un alto nivel de conocimientos por parte de la madre en el área del cuidado de la cavidad oral influye de forma importante en el desarrollo de buenos hábitos de higiene por parte de sus hijos y en la obtención exitosa de denticiones saludables. Del mismo modo, la Academia Americana de Odontología Pediátrica asegura que la responsabilidad de la higiene oral, para que sea efectiva en la prevención de la caries dental, es, en principio, de los padres, mientras el niño desarrolla sus habilidades cognitivas y motrices [21].

En este sondeo se encontraron bajos niveles de conocimiento en salud oral por parte de los acudientes de menores de 5 años, teniendo en cuenta la totalidad de la muestra, lo cual evidencia el aumento del riesgo de aparición de nuevas lesiones de caries y la exacerbación de las ya existentes.

Un hallazgo de esta investigación fue que el conocimiento previo con que contaban los acudientes acerca de la utilidad del flúor en la prevención y el tratamiento de la caries dental tenía bastante probabilidad de reducir la aparición de caries en los niños menores de 5 años. Este resultado está de acuerdo con las conclusiones hechas por varias publicaciones, en las que se asevera que el uso de flúor tópico ayuda a la prevención, inhibición y reparación de la caries [22-24].

Para el continente americano, el promedio de dientes cariados, obturados y extraídos por caries en el 2004 fue de tres dientes [9]; este valor es menor que el encontrado en la presente investigación (4,7 dientes). Se debe tener en cuenta que la media de severidad de caries en América se dio como resultado de aplicar el índice COP a niños de 12 años de edad que estuvieron expuestos a factores de riesgo durante aproximadamente ocho años, más que el promedio de los niños participantes en el presente estudio. Este valor incrementado puede estar relacionado con lo descrito por Álvarez et al., que definieron el periodo de 3 a 5 años de edad como una época en la que los niños no tienen suficiente destreza manual para hacer la limpieza de la cavidad oral por sus propios medios y dependen de sus padres para tal fin [19].

La mayor parte de los acudientes entrevistados eran las madres de los niños evaluados, hallazgo que es importante, teniendo en cuenta lo encontrado por Åstrøm, quien sostuvo que dentro del grupo familiar, la madre tiene un papel destacado en el estado de salud y los hábitos de higiene de los niños [25]. Esta situación puede ser ventajosa a la hora de transmitir de forma exitosa la información para el correcto cuidado de la cavidad oral, como lo registran Weinstein et al. en su investigación, quienes encontraron que, mediante la consejería motivacional dirigida a las madres de familia y un seguimiento a esta durante un año, se puede reducir la probabilidad de que a sus hijos les aparezcan lesiones asociadas a la caries $(\mathrm{OR}=0,35$; IC $95 \%=0,15-0,83)$ [26].

La baja escolaridad de los acudientes del grupo de los casos que participaron en este estudio y la alta prevalencia de caries en los menores de 5 años del mismo grupo es coherente con lo reportado por estudios anteriores, que vinculan bajos niveles de educación de los padres de familia con altos niveles de caries dental en sus hijos [9].

Las personas con bajo nivel educativo son catalogadas como población en situación de desventaja y suelen cargar con la mayor parte de las enfermedades de la cavidad oral [1]. Sin embargo, en la presente investigación no hubo diferencias entre los grupos estudiados con respecto al nivel educativo; en ambos, el $70 \%$ de los participantes encuestados estudió hasta la básica primaria.

Asimismo, fue un hallazgo de esta investigación que, comparado con estudios anteriores $[11,20]$, una mayor proporción de cuidadores de niños aseguraron que la caries dental se producía por mala higiene oral y por un consumo excesivo de alimentos azucarados. De igual forma, la restricción de alimentos dulces fue considerada como una medida adecuada para prevenir la aparición de caries dental, como se encontró en investigaciones previas [20,27]. No obstante, en la presente investigación no se evidenciaron diferencias entre los grupos de casos y controles con respecto a los hallazgos mencionados.

Por otra parte, hubo diferencias, entre los grupos evaluados, en lo concerniente a la apreciación que tuvieron los acudientes de la lesión inicial de la caries. El $11 \%$ de los acudientes dentro del grupo control identificó cómo se veía la caries inicialmente, comparado con el $3 \%$ de los acudientes del grupo de los casos $(\mathrm{OR}=3,31 ;$ IC $95 \%=0,95-11,55)$. Este hallazgo es congruente con lo relacionado por Chhabra y Chhabra, que concluyeron que la falta de conocimientos, acerca del cuidado de los dientes, por parte de los padres de familia, crea barreras en el desarrollo de actividades preventivas contra la caries dental [28].

Conocer el estadio en el que se encuentre la caries otorga una ventaja sobre la manera como se puede abordar el tratamiento de la lesión. Sin embargo, la metodología de la encuesta realizada en esta investigación fue solo teórica, sin un componente práctico que verificará la verdadera capacidad de un tutor para identificar la apariencia inicial de una caries. En general, las campañas educativas en cualquier área de la salud ofrecen a los usuarios cantidades de información, charlas, documentos y videos relacionados con un tema, pero no llevan a cabo tareas de retroalimentación o seguimiento 
a ese aprendizaje, ni se mide la adherencia al programa educativo.

La asistencia al odontólogo como medida para prevenir la caries fue una aseveración común en los dos grupos encuestados y guarda resultados similares con otros estudios, donde se prioriza, por los entrevistados, la presencia del odontólogo como recurso curativo de la caries $[11,20,24]$.

Ahora bien, una importante cantidad de flúor se encuentra en las cremas dentales (1500 partes por millón, aproximadamente). La aplicación de flúor sobre las superficies dentales es de gran ayuda para remineralizar lesiones incipientes, inhiben la actividad enzimática de los microorganismos de la placa e incluso pueden cambiar la composición de la microflora de la superficie dentaria. La combinación del flúor y la adecuada remoción de la placa controlan de manera efectiva la caries dental [26]. En el presente estudio, son bajos los niveles de conocimiento sobre las funciones del flúor para prevenir la caries dental, tanto en los casos, como en los controles, sin mostrar diferencias significativas entre ambos. Esto tiene similitud con lo encontrado por Franco et al., donde el $14 \%$ de un grupo de madres encuestadas, clasificadas en varios estratos socioeconómicos, afirmó saber para qué servía el flúor [10].

La edad promedio en los niños del grupo casos fue de 3,7 años. La existencia de caries en estos niños puede sugerir que fueron llevados a consulta odontológica de forma tardía, conducta que no sigue las recomendaciones de la Academia Americana de Pediatría, la cual menciona que un niño debe ser evaluado por un profesional de la odontología a partir de 1 año de edad o antes, esto con el fin de identificar factores de riesgo individuales para la formación de caries. Esta revisión debe hacer parte de una estrategia intensa para prevenir la aparición de caries en edad preescolar, que incluye la asesoría anticipada para las futuras madres que asisten a los controles prenatales y modificación en las actividades de alimentación y técnicas de higiene que perjudiquen la salud oral de los niños [29].

Este hallazgo puede estar relacionado con la creencia de los prestadores de los servicios de salud que todavía operan con base en la norma técnica 412 del 2000, que refiere que las actividades de promoción de la salud oral y prevención de las enfermedades de la boca deben realizarse en pacientes a partir de los dos años de edad [30].

Diez años después de expedir las guías para la atención en actividades de promoción y prevención en salud, el Ministerio de la Protección Social expide la Circular 034 de 2010, donde se solicita a las entidades que prestan el servicio de salud oral favorecer el acceso a los servicios de odontología a los menores de 1 año, para poder diagnosticar y tratar de manera temprana la caries no cavitacional, con el fin de evitar la formación defectuosa de los dientes permanentes [31].

Concluyeron los autores del ENSAB IV que los problemas de salud oral encontrados deben ser atribuidos, en mayor medida, a la indolencia de las personas frente al cuidado de su boca, hallando, igualmente, dificultades para acceder al servicio de salud [20]. Sin embargo, la población objeto de esta investigación tiene facilidades de acceso a los servicios de salud. Además de contar con institución de salud cercana a su residencia y recibir atención de forma gratuita, existen programas con enfoques multidisciplinarios donde se recibe atención por parte del personal médico, de enfermería y odontológico, con especial énfasis en atender mujeres gestantes y niños en edades entre 0 y10 años. La atención es óptima; no obstante, se requiere de un mayor acercamiento, por fuera del consultorio, a las familias. Ahora bien, de acuerdo con los hallazgos del presente estudio, la caries dental de los niños menores de 5 años puede atribuirse al desconocimiento de las buenas prácticas de higiene oral y las consecuencias que esto pueda ocasionarles.

\section{Limitaciones}

No se confirmó que los acudientes que asistieron con los niños a consulta odontológica fueran los cuidadores principales, el tiempo efectivo de cuidado que tenían estos hacia los niños y si compartían esta actividad de cuidado con otros miembros de la familia o habitantes del hogar.

La persona que llevó a cabo el proceso de recolección de información fue el investigador principal, quien, además, era el mismo odontólogo que atendía a los usuarios en el Centro de Salud Santo Domingo Savio. Esto pudo haber limitado las respuestas de los entrevistados, llevándolos a responder positiva o negativamente ítems que no correspondían a la realidad.

Otra limitante fue no haber registrado las lesiones incipientes o iniciales de caries dental. Aunque el tratamiento de una lesión inicial de caries difiere respecto al de la lesión cavitaria, el origen de ambas lesiones es similar y los factores de riesgo son los mismos.

De igual modo, la selección de la muestra se realizó con personas que acceden al servicio de salud, utilizando un muestreo no probabilístico, y sin ser representativos del área geográfica de cobertura del centro de salud.

\section{Conclusiones}

En esta investigación se describen los conocimientos que sobre higiene oral tienen los acudientes responsables de menores de 5 años que consultaron en un centro de salud de la ciudad de Medellín, con el fin de identificar en qué aspectos hay falencias y así poder sugerir 
mejoras en la educación brindada a los usuarios de los servicios de salud que tienen bajo su cargo infantes en edad preescolar.

En este estudio se encontraron diferencias significativas entre los grupos casos y controles acerca de cómo aprecian los acudientes una lesión inicial de caries. Identificar las lesiones de caries a tiempo evita tratamientos invasivos e irreversibles, mejorando así la calidad de vida de los individuos, en este caso, la de los niños menores de 5 años.

Los resultados sugieren la necesidad de realizar proyectos de investigación que busquen la forma de comprobar, de un modo más tangible y verificable, los conocimientos que sobre higiene oral poseen los responsables de los niños menores de 5 años, y así comprender, de mejor manera, su influencia sobre la prevalencia de la caries dental en este grupo poblacional, así como para diseñar estrategias de promoción y prevención que permitan la disminución de la incidencia de la caries.

Se requiere construir espacios de comunicación e interacción entre los profesionales de la salud y los usuarios, que permitan la transformación de sus saberes, y promuevan el autocuidado y la detección temprana de la caries dental.

Para generar acciones educativas oportunas y con impacto, se debe dar inicio a estas desde el momento de la gestación humana y mediante un trabajo interdisciplinar que faculte una visión holística de familia y salud. A su vez, es importante establecer procesos de continuidad respecto a estas acciones, asegurando el acompañamiento y la educación en salud bucal durante varias etapas del desarrollo del ser humano.

La utilización de medios de comunicación, el uso de redes sociales y diferentes estrategias de información, educación y comunicación (IEC), y de movilización social, se convierten en un factor aliado para las actividades educativas masivas. Para ello, es necesario evaluar los contenidos que se transmiten y plantear estrategias innovadoras que permitan una mayor aprehensión del conocimiento.

\section{Agradecimientos}

Este proyecto fue posible gracias al apoyo y la ayuda incondicional de Sara Agudelo, Viviana Álvarez y de los participantes que dedicaron tiempo para nutrir esta investigación con sus respuestas.

\section{Conflictos de interés}

Esta investigación tuvo el único fin de generar nuevo conocimiento y buscar el beneficio de la comunidad. Ningún autor tuvo conflicto de interés.

\section{Fuentes de financiación}

Todos los gastos fueron asumidos por los investigadores. Este artículo fue derivado de un trabajo de grado universitario para obtener el título de magíster en Epidemiología, obtenido en agosto de 2015 en la Universidad CES en Medellín, Colombia, sin aporte económico de ninguna institución.

\section{Referencias}

1 Petersen PE, Bourgeois D, Ogawa H, Estupinan-Day S, Ndiaye C. The global burden of oral diseases and risks to oral health. Bull World Health Organ. 2005;83(9):661-9.

2 Sarumathi T, Saravana Kumar B, Manjula Datta, Hemalatha VT, Aarthi Nisha V. Prevalence, severity and associated factors of dental caries in 3-6 year old children. J Clin Diagn Res JCDR [Internet]. Agosto 2013 [citado 2015 jun. 9]; 7(8):1789-92. Disponible en: http://www.jcdr.net/article fulltext.asp?issn=0973-709x\& year $=2013 \&$ volume $=7 \&$ issue $=8 \&$ page $=1789 \&$ issn $=0973$ $709 x \&$ id $=3277$

3 Selwitz RH, Ismail AI, Pitts NB. Dental caries. Lancet. 2007;369(9555):51-9.

4 Acs G, Shulman R, Ng MW, Chussid S. The effect of dental rehabilitation on the body weight of children with early childhood caries. Pediatr Dent. 1999; 21(2):109-13.

5 Reisine ST. The impact of dental conditions on social functioning and the quality of life. Annu Rev Public Health. 1988;9:1-19.

6 Low W, Tan S, Schwartz S. The effect of severe caries on the quality of life in young children. Pediatr Dent. 1999;21(6):325-6.

7 Riojas-Garza MT. Anatomía dental. 2. ${ }^{a}$ ed. México: Manual moderno; 2009. p. 204.

8 Escobar G, Ortiz A, Mejía LM. Caries dental en los menores de veinte años en Colombia: un problema de salud pública. Rev. Fac. Nac. Salud Pública. 2003;21(2):107-118.

9 Petersen PE. Sociobehavioural risk factors in dental caries - international perspectives. Community Dent Oral Epidemiol. 2005;33(4):274-9.

10 Franco AM, Jimenez J, Saldarriaga C, Zapata L, Saldarriaga A, Martignon $\mathrm{E}$, et al. Conocimientos y prácticas de cuidado bucal de las madres de niños menores de 6 años. Rev CES Odontol. 2003;16(1):27-33.

11 Rodríguez N, Valencia A, Díaz P. Promoción de la salud y sistema de salud colombiano: reflexiones sobre la formación de profesionales. Hacia Promoc Salud. 2013;18(1):26-40.

12 Eslava JC. Repensando la promoción de la salud en el Sistema General de Seguridad Social en Salud. Rev Salud Pública. 2006;8(Sup. 2):106-15.

13 Benavente L, Chein S, Campodónico C, Palacios E, Ventocilla MS, Castro A. Nivel de conocimientos en salud bucal de las madres y su relación con el estado de salud bucal del niño menor de cinco años de edad. Odontol Sanmarquina. 2012;15(1):14-18.

14 National Research Council, National Academy of Sciences. A survey of the literature of dental caries [Internet]. Books on Demand; Washington, 1984 [citado 2018 abr. 1]. Disponible en: https://books. google.com.co/books?id=K0wrAAAAYAAJ\&pg=PA509\&lpg= PA509\&dq $=\mathrm{A}+$ survey + of + the + literature + of + dental + caries $\&$ sou 
rce $=$ bl\&ots $=$ GDAGnJ1yef\&sig=zIvqGDdMLFNGORSaUMekd

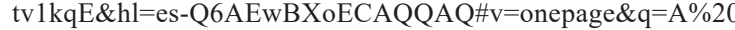
survey $\% 20$ of $\% 20$ the $\% 201$ iterature $\% 20$ of $\% 20$ denta $1 \% 20$ caries $\& \mathrm{f}=$ false

15 Organización Panamericana de la Salud. Direccion xeral de innovacion e xestion de saúde publica. Epidat 3.1. Xunta de Galicia; 2006.

16 Martignon S, Bautista-Mendoza G, González-Carrera MC, Lafaurie-Villamil G. Instrumentos para evaluar conocimientos, actitudes y prácticas en salud oral para padres/cuidadores de niños menores. Rev. Salud Pública. 2008;10(2):308-14.

17 Colombia, Ministerio de Salud. Resolución 8430 de 1993, Por la cual se establecen las normas científicas, técnicas y administrativas para la investigación en salud.

18 Saied-Moallemi Z, Virtanen JI, Ghofranipour F, Murtomaa H. Influence of mothers' oral health knowledge and attitudes on their children's dental health. Eur Arch Paediatr Dent. 2008;9(2):79-83.

19 Álvarez AM, Cuartas JC, Maya AM, Cárdenas JM, Arias MI, Jaramillo A. Relación entre percepción de los padres ante el tratamiento odontológico y sus hábitos de higiene oral, con la historia de caries dental en sus hijos entre 3 y 5 años de edad. Rev CES Odontol. 2002;15(1):13-18.

20 Colombia, Ministerio de Salud (Minsalud). IV Estudio Nacional de Salud Bucal. ENSAB Iv. Metodología y determinacion social de la salud bucal. [Internet]. 2014. [citado 2015 jun. 9] Disponible en: http://www.minsalud.gov.co/sites/rid/Lists/BibliotecaDigital/ RIDE/VS/PP/ENSAB-IV-Metodologia.pdf

21 American Academy of Pediatric Dentistry. Guideline on cariesrisk assessment and management for infants, children, and adolescents. Pediatr. Dent. 2013;35(5):E157-64.

22 Liu BY, Lo ECM, Chu CH, Lin HC. Randomized trial on fluorides and sealants for fissure caries prevention. J Dent Res. 2012;91(8):753-8
23 Adair SM. Evidence-based use of fluoride in contemporary pediatric dental practice. Pediatr Dent. 2006; 28(2):133-42; discussion 192-198.

24 Horowitz HS. The $2001 \mathrm{CDC}$ recommendations for using fluoride to prevent and control dental caries in the United States. J Public Health Dent. 2003;63(1):3-8; discussion 9-10.

25 Åstrøm AN. Parental influences on adolescents' oral health behavior: Two-year follow-up of the Norwegian Longitudinal Health Behavior Study participants. Eur J Oral Sci. 1998;106(5):922-30.

26 Weinstein P, Harrison R, Benton T. Motivating parents to prevent caries in their young children: One-year findings. J Am Dent Assoc. 1939. 2004;135(6):731-8.

27 Franco AM, Santamaría A, Kurzer A, Castro L, Giraldo M. El menor de seis años: situación de caries y conocimientos y prácticas de cuidado bucal de sus madres. Rev CES Odontol. 2004;17(1):1929.

28 Chhabra N, Chhabra A. Parental knowledge, attitudes and cultural beliefs regarding oral health and dental care of preschool children in an Indian population: A quantitative study. Eur Arch Paediatr Dent. 2012;13(2):76-82.

29 American Academy of Pediatric Dentistry Council on Clinical Affairs. Policy on early childhood caries (ECC): unique challenges and treatment options. Pediatr Dent. 2005-2006;27(7 Suppl):34-5.

30 Colombia, Ministerio de Salud (Minsalud). Norma técnica para la atención preventiva en salud oral. Bogotá; 2000.

31 Colombia, Ministerio de la Protección Social, Viceministerio de Salud y Bienestar. Circular 034 de 2010. "Acciones para el mejoramiento de la salud bucal" (2010 jun 23). 